BULL. AUSTRAL. MATH. SOC.

VOL. 19 (1978), 138.

\title{
Stably layered fluids
}

\section{A.K. Laing}

Various problems associated with a stably layered fluid are studied.

Firstly, the flow of a layered fluid from a reservoir is considered. Steady solutions are sought and found to depend on the flow at the critical points, that is, points at which the long wave speeds for the different modes of gravity wave propagation vanish. For certain distributions of the layers' depths it is found that there are too many critical points, and hence the flow is overdetermined and no steady solutions are possible. Thus, when reservoir conditions are slowly altered so as to pass from one depth ratio to another, the flow will not necessarily change slowly too.

A closed three layered fluid with small density differences between the layers is also studied. The nonlinear interactions between the two, closely related, internal wave modes are investigated, and general evolution equations are obtained for small disturbances at the interfaces. Permanent solutions involving one mode alone are calculated, but it is found that at certain wavelengths the slower mode permanent waves resonantly generate harmonics of the faster mode.

When the group velocity of a train of faster mode waves is the same as the phase velocity of a slower mode wave there is significant interaction leading to a permanent wave structure comprising a group of permanent envelope and a carrier wave also of permanent shape. For each wavelength the theory predicts several forms of structure. However, numerical solutions were only obtained for some of the simpler forms.

Received $25 \mathrm{July}$ 1978. Thesis submitted to the University of Canterbury, June 1978. Degree approved, July 1978. Supervisor: Dr P.J. Bryant. 